\title{
IMPACTO DEL LABORATORIO VIRTUAL EN EL APRENDIZAJE POR DESCUBRIMIENTO DE LA CINEMÁTICA BIDIMENSIONAL EN ESTUDIANTES DE EDUCACIÓN MEDIA
}

\author{
IMPACT OF VIRTUAL LABORATORY IN DISCOVERY LEARNING OF TWO- \\ DIMENSIONAL KINEMATICS IN SECONDARY EDUCATION STUDENTS
}

\begin{abstract}
Gustavo Adolfo Angulo Mendoza; gangulo@unab.edu.co
Leónidas Onésimo Vidal Espinosa; o-vidal@hotmail.com

Gabriela García Ortiz; gabriela.garcia.ortiz@itesm.mx
\end{abstract}

Tecnológico de Monterrey

\begin{abstract}
RESUMEN
Este artículo resume un estudio cuyo propósito fue determinar la incidencia del laboratorio virtual en el aprendizaje de la cinemática bidimensional, para un contexto específico. El estudio es de tipo cuantitativo, con diseño cuasiexperimental, pre-prueba, pos-prueba y grupos intactos, uno de ellos de control. Las pruebas paramétricas permitieron concluir que, en las dimensiones afectiva y cognitiva, existía diferencia significativa a favor de los alumnos que emplearon una estrategia basada en el uso del laboratorio virtual. Para la dimensión procedimental no se encontró diferencia estadísticamente significativa.
\end{abstract}

PALABRAS CLAVE: Enseñanza de la física, laboratorio virtual, simulador, cinemática, aprendizaje por descubrimiento, software educativo, tecnología educativa.

\begin{abstract}
This article summarizes a study whose objective was to determine the incidence of virtual laboratory in the learning of two-dimensional kinematics to a specific context. The study is quantitative, quasi-experimental designed, with pre-test, post-test and intact groups, one control. Parametric tests led to the conclusion that in the affective and cognitive dimensions, there was significant difference in favor of students who used a strategy based on the use of virtual laboratory. For the procedural dimension, there was no statistically significant difference.
\end{abstract}

KEY WORDS: Physics teaching, virtual laboratory, simulator, kinematics, discovery learning, educational software, educational technology. 


\section{INTRODUCCIÓN}

\subsection{Marco contextual.}

Los participantes del estudio fueron estudiantes de décimo grado de Educación Media Académica de una institución educativa ubicada en la ciudad de Cartagena de Indias, departamento de Bolívar, República de Colombia. La institución es de carácter público, y presta el servicio educativo desde el nivel preescolar hasta el nivel medio. Los participantes provienen de familias con un nivel socioeconómico medio y medio-bajo.

El estudio se sitúa en la enseñanza de la Física, concretamente, en el aprendizaje de la cinemática bidimensional. Este tema específico hace parte de la mecánica clásica, donde se estudian diversos eventos relacionados con el movimiento. En el nivel de Educación Media se busca que los estudiantes puedan hacer análisis cuantitativos y cualitativos de estos eventos.

\subsection{Antecedentes del problema.}

El plantel donde se llevó a cabo el estudio ha tenido un buen desempeño en las pruebas SABER 11, implementadas por el ICFES, alcanzando el nivel de desempeño Superior durante los últimos diez años. Sin embargo, la institución no ha alcanzado la meta de ubicarse en la máxima categoría de desempeño: el nivel Muy Superior. Específicamente en la asignatura de física, el desarrollo de competencias en la prueba se ubica en el nivel medio. En ese sentido los docentes y directivos del plantel han buscado estrategias para mejorar la calidad de los aprendizajes. Una de esas estrategias es el uso de tecnologías de la información y la comunicación (TIC) en los procesos de enseñanza. Es importante hacer notar que varios estudios (Bayrak, 2008; Catalán, Serrano y Concari, 2010; Debel, Cuicas, Casadei, y Álvarez, 2009) han demostrado la eficacia de estas herramientas en otros niveles de educación.

\subsection{Planteamiento del problema.}

Se pretendió dar respuesta a la siguiente pregunta, ¿Cuál es el impacto del laboratorio virtual (LV) en el aprendizaje por descubrimiento de la cinemática bidimensional en estudiantes de décimo grado de Educación Media? De esta pregunta principal se derivan las siguientes preguntas:

Para el grado décimo de Educación Media, ¿existe relación entre la utilización de un LV y la actitud de los estudiantes hacia el aprendizaje de la cinemática bidimensional?

¿Existe diferencia estadísticamente significativa en el nivel de comprensión de la cinemática bidimensional, entre estudiantes que reciben instrucción mediada por un LV y estudiantes que reciben instrucción tradicional?

¿Existe relación entre el uso del LV y el desarrollo de habilidades para resolver problemas de física? 


\subsection{Objetivos.}

El objetivo general del presente estudio es determinar el impacto del LV en el aprendizaje por descubrimiento de la cinemática bidimensional en estudiantes de décimo grado de Educación Media.

A partir de este objetivo se plantean los siguientes objetivos específicos:

(a) Establecer la relación entre la utilización del LV y la actitud de los estudiantes de décimo grado de Educación Media hacia el aprendizaje de la cinemática bidimensional;

(b) Determinar si existe diferencia en el nivel de comprensión de los principios de la cinemática bidimensional, entre estudiantes que reciben instrucción mediada por un LV y estudiantes que reciben instrucción tradicional, para el décimo grado de Educación Media; $y$,

(c) Establecer la relación entre el uso del LV y el desarrollo de habilidades para resolver problemas de física, en el décimo grado de Educación Media.

\subsection{Justificación.}

Este estudio aporta evidencia empírica sobre la incidencia de los LV en la enseñanza de la física en el nivel de Educación Media. Existen importantes estudios sobre el tema pero orientados hacia otros niveles de educación (García y Gil, 2006; Esquembre, 2005; Kofman, Catalán y Concari, 2004; Giorgi, Cámara y Kofman, 2004; Fogliati, Catalán y Concari, 2004; Casadei, Cuicas, Debel y Álvarez, 2008). Asimismo, existen estudios enfocados a ámbitos de la física diferentes a la cinemática bidimensional (Becerra, 2005; Amaya, 2008).

Esta investigación podría beneficiar a los diferentes actores del proceso educativo: docentes, directivos docentes, investigadores y autoridades educativas. Los resultados de esta investigación les permitirían tomar importantes decisiones sobre la inclusión de herramientas TIC como medios para favorecer el aprendizaje de la física en el nivel de Educación Media.

\subsection{Limitaciones del estudio.}

El estudio se desarrolló durante el periodo académico correspondiente al año 2011. En el primer semestre se adelantó el componente conceptual de la investigación (planteamiento, marco teórico y metodología), dejando para el segundo semestre el trabajo de campo. Esta importante restricción de tiempo obligó a delimitar el estudio, enfocándolo en el uso de una herramienta determinada (laboratorio virtual), con una estrategia pedagógica definida (aprendizaje por descubrimiento), en una parte específica de la física (cinemática bidimensional), para un nivel específico de enseñanza (décimo grado de Educación Media). En ese sentido, para cumplir con los plazos de las entregas parciales, fue necesario seleccionar un diseño metodológico adecuado que permita contar con resultados concretos en poco tiempo.

De otro lado, es importante hacer notar que los hallazgos de esta investigación no pueden ser generalizados a otros contextos de aprendizaje; primero, por el tipo de muestra elegida para desarrollar el estudio, y segundo, por el propio diseño de investigación realizado. 


\subsection{El Laboratorio Virtual.}

EI LV hace parte de una categoría de software que permite hacer simulaciones de experimentos, obteniendo resultados que serían muy difíciles de conseguir si se trata de resolver el modelo matemático (Kowalski, 1985). Entre las ventajas del uso del LV, Sierra (2000) destaca:

- Es posible recrear fenómenos cuya reproducción sería improbable en un ambiente escolar.

- Favorece el contraste de las ideas previas.

- Es posible manipular las variables del modelo lo que favorece el aprendizaje por descubrimiento.

- Le permite al alumno concentrarse en los principios físicos que intervienen en el fenómeno y no sólo en los procedimientos matemáticos.

La figura 1 muestra una captura de pantalla de un experimento virtual sobre cinemática bidimensional.

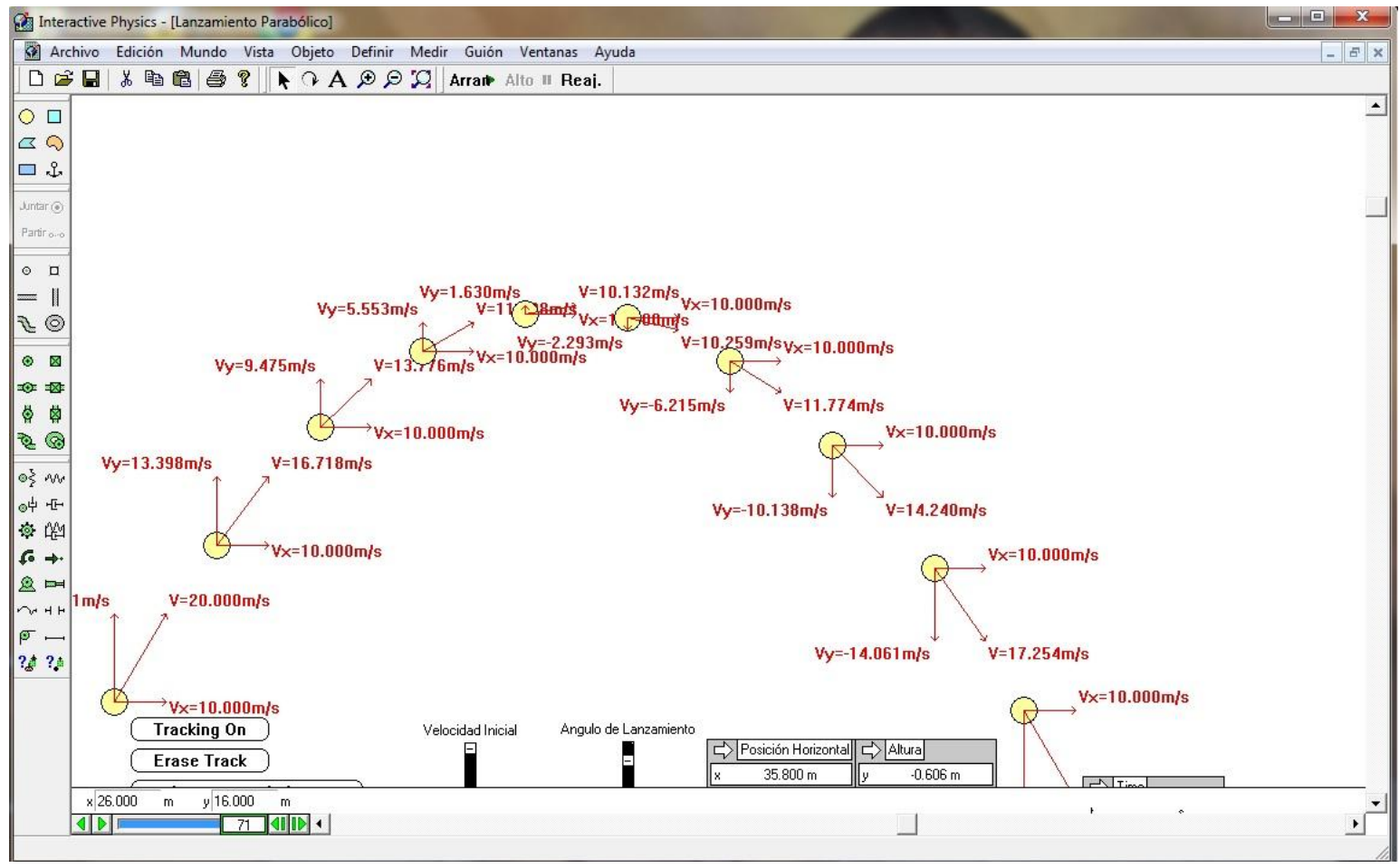

Figura 1. Experimento virtual sobre lanzamiento parabólico. Desarrollado en Interactive Physics ${ }^{\circledR}$.

Uno de los efectos más notables de la utilización del LV es la adopción de un nuevo perfil docente. El profesor asume un rol de facilitador y orientador, y entiende que debe ser el alumno el protagonista del proceso (García y Gil, 2006). En esa línea, se pueden mencionar las siguientes funciones del profesor en su nuevo rol:

- Proveedor de recursos.

- Organizador.

- Tutor. 
- Investigador.

- Facilitador.

En ese sentido, el desarrollo de actividades utilizando el LV involucra diversos niveles de abstracción que son usados en una estrategia instruccional de cuatro fases: motivación, investigación, formalización y transferencia (Sierra, 2005).

\subsection{El Aprendizaje por Descubrimiento.}

El modelo didáctico de aprendizaje por descubrimiento establece que la mejor forma en que los estudiantes aprenden ciencia es, sencillamente, haciéndola. En ese sentido, la formación en ciencias debe fundamentarse en experiencias que le ofrezcan al alumno la oportunidad de recrear los descubrimientos científicos. Esta concepción se basa en la premisa de que el alumno desarrolla operaciones intelectuales semejantes a las del científico, y por tanto, aplicará las fases del método científico en su proceso de indagación (Kelly, 1955).

Una de las funciones del docente es la de propiciar un contexto que favorezca el descubrimiento, generando preguntas detonantes o problemas que los estudiantes deban resolver. De esta manera, una secuencia didáctica basada en aprendizaje por descubrimiento, consta de cinco fases: presentación del problema, identificación de variables y recolección de datos, experimentación, organización e interpretación de resultados y reflexión (Escribano, 2008). El modelo didáctico de aprendizaje por descubrimiento se complementa otras estrategias para la enseñanza de las ciencias como: aprendizaje a través del conflicto cognitivo, enseñanza por investigación dirigida y enseñanza por contrastación de modelos.

\subsection{Investigaciones Empíricas Relacionadas.}

Los estudios consultados (Amaya, 2008; Bayrak, 2008; Becerra, 2005; Chou, 1998; Sierra, 2005; Tüizüz 2010) buscaban determinar la incidencia del LV y, en general, del software de simulación, en el aprendizaje de diversos ámbitos de la física en diferentes niveles de educación. Estas investigaciones se desarrollaron desde el enfoque cuantitativo, siguiendo un diseño cuasiexperimental con pre-prueba, post-prueba y grupos intactos. Se utilizó estadística descriptiva para analizar los datos recogidos y pruebas paramétricas para determinar si existía diferencia significativa entre los resultados del grupo experimental y el grupo de control.

En esos trabajos se recomiendan futuros estudios como: impacto un diseño instruccional basado en el uso de LV en la enseñanza de áreas diferentes a la mecánica, incidencia del LV en diversos niveles educativos, impacto de las representaciones de docente y alumnos sobre el aprendizaje por investigación mediado por LV, y, la aplicación de la estrategia de aprendizaje por investigación a través de LV en la solución de problemas interdisciplinares próximos a la realidad. 


\section{METODOLOGÍA}

La investigación se abordó desde el enfoque cuantitativo, con un alcance correlacional y siguiendo un diseño cuasiexperimental con preprueba, posprueba y grupos intactos, tal como se muestra en la tabla 1.

\begin{tabular}{llll}
\hline \multirow{2}{*}{ Grupos } & $\begin{array}{l}\text { Medida de } \\
\text { la preprueba }\end{array}$ & $\begin{array}{l}\text { Tratamiento } \\
\text { experimental }\end{array}$ & $\begin{array}{l}\text { Medida de } \\
\text { la posprueba }\end{array}$ \\
\hline $\mathrm{G}_{1}$ & $\mathrm{O}_{1}$ & $\mathrm{X}$ & $\mathrm{O}_{2}$ \\
$\mathrm{G}_{2}$ & $\mathrm{O}_{3}$ & - & $\mathrm{O}_{4}$ \\
\hline
\end{tabular}

Tabla 1. Diseño cuasiexperimental con preprueba, posprueba y grupo de control.

Este enfoque permitió medir el efecto que tiene sobre las variables dependientes, la manipulación intencional de la variable independiente (utilización de software de laboratorio virtual). En la literatura consultada, se encontraron varios estudios afines (Amaya, 2008; Amaya 2009; Casadei et al., 2008; Debel et al., 2009; Olivero y Chirinos, 2007; Rodríguez, Mena y Rubio, 2009; Sierra, 2005) que también fueron desarrollados siguiendo un diseño cuasiexperimental.

Tal como se precisó previamente, es necesario resaltar que los hallazgos de este estudio no pueden ser extrapolados a otros contextos educativos, tanto por el tipo de muestra elegida como por el propio diseño de investigación realizado.

La población estaba conformada por todos los estudiantes de décimo grado de la institución educativa seleccionada. A partir de esta población caracterizada, se procedió a determinar el tamaño de la muestra con el programa STATS ${ }^{\circledR}$. Para un error estándar de $1 \%$ el programa arrojó un tamaño de la muestra de 56. Se estableció que el grupo experimental tendría 27 alumnos y el grupo de control 29. La tabla 2 muestra algunas características de los grupos participantes.

\begin{tabular}{lllll}
\hline Grupos & $\begin{array}{l}\text { Promedio } \\
\text { de edad }\end{array}$ & $\begin{array}{l}\text { Número de } \\
\text { Hombres }\end{array}$ & $\begin{array}{l}\text { Número de } \\
\text { Mujeres }\end{array}$ & $\begin{array}{l}\text { Estudiantes } \\
\text { Repitentes }\end{array}$ \\
\hline $\mathrm{G}_{1}$ (Experimental) & 15,58 & 16 & 11 & 2 \\
$\mathrm{G}_{2}$ (Control) & 15,39 & 19 & 10 & 0 \\
\hline
\end{tabular}

Tabla 2. Características generales de los grupos participantes en el estudio.

Las tres variables dependientes que se analizaron fueron:

a) Actitud de los estudiantes hacia la ciencia, los conocimientos científicos y las asignaturas de ciencias,

b) nivel de comprensión de los principios de la cinemática bidimensional, y,

c) nivel de desarrollo de habilidades para resolver problemas de física.

Estas variables fueron medidas, respectivamente, a través de: el test para diagnosticar la actitud de los estudiantes hacia la ciencia (Penichet y Mato, 1999), la prueba estandarizada sobre los conceptos y principios de la cinemática bidimensional y la prueba de solución de problemas relacionados con la cinemática bidimensional. La fiabilidad y validez de estos instrumentos fueron validadas a través de pruebas piloto donde se calcularon coeficientes de confiabilidad. 
El estudio se desarrolló en tres etapas:

- La fase pre-instruccional donde se llevó a cabo la selección de la muestra y designación de los grupos, la solicitud de autorización y consentimientos informados, las pruebas piloto, la aplicación de la preprueba, la validación de la equivalencia inicial de los grupos y la contextualización.

- La fase instruccional, donde se desarrollarán las secuencias didácticas respectivas para cada grupo.

- La fase post-instruccional, en la cual se aplicarán las pospruebas y el análisis de datos.

Para el análisis de los datos, se aplicó estadística descriptiva y pruebas paramétricas mediante el uso del Paquete Estadístico para las Ciencias Sociales (Statistical Package for the Social Sciences o SPSS ${ }^{\circledR}$ de IBM). El análisis descriptivo incluye el cálculo de media y desviación estándar. De otro lado, el cálculo de la prueba t-Student permitió establecer si el grupo experimental y el grupo de control diferían entre sí de manera significativa respecto de las puntuaciones medias de las prepruebas y las pospruebas.

\section{RESULTADOS}

\subsection{Comprobación de la validez y fiabilidad de los instrumentos.}

Los instrumentos empleados fueron revisados por cinco expertos quienes emitieron un concepto favorable. Se comprobó la fiabilidad y la validez de cada instrumento. Respecto al test de Penichet y Mato para evaluar la actitud hacia la ciencia, el coeficiente de Spearman-Brown entre los ítems pares e impares arrojó un resultado de 0,256, que constituye una correlación significativa al nivel 0,01. El cálculo de los coeficientes de Pearson entre los ítems del test mostró que un 52\% tiene una correlación significativa. El análisis factorial concluyó que existen cuatro factores que explican un $65 \%$ de la varianza total de las respuestas al test.

Para la prueba de conceptos de cinemática bidimensional, el coeficiente KR-20 arrojó un valor de 0,770 , que muestra una correlación significativa. Del cálculo del coeficiente de Pearson entre los ítems de la prueba, se concluye que un 30\% muestra una correlación significativa. El análisis factorial permite deducir que existen ocho factores que explican un $81 \%$ de la varianza total de las respuestas a la prueba. Asimismo, para validar la fiabilidad intraevaluadora de la prueba de solución de problemas, se le pidió a un experto que calificara las mismas pruebas en dos momentos diferentes. El coeficiente de Pearson entre las calificaciones del primer y del segundo momento fue de 1,00 que es significativo a 0,01. Para comprobar la fiabilidad interevaluadora, dos docentes calificaron simultáneamente las mismas pruebas. El coeficiente de Pearson entre las calificaciones de los dos evaluadores fue de 0,945 que es significativo a 0,01.

\subsection{Situación de los estudiantes en la fase pre-instruccional.}

A partir de los datos de la tabla 3, se realizó la prueba t, que arrojó un valor de 0,287. Este valor es inferior al que se muestra en la tabla de la distribución t de Student a un nivel de confianza de 0,05 y con 60 grados de libertad (valores de referencia para todas las pruebas $t$ realizadas). 


\begin{tabular}{lll}
\hline Medida & GE & GC \\
\hline Media & 48,65 & 48,04 \\
Desviación estándar & 7,42 & 6,97 \\
\hline
\end{tabular}

Tabla 3. Resultados del pretest de pretest de Penichet y Mato.

Se determinó que, antes de la ejecución de las respectivas secuencias didácticas, no existía diferencia estadísticamente significativa entre la media en el test de actitud del grupo experimental (GE) y del grupo de control (GC).

De manera similar, la tabla 4 condensa los resultados obtenidos por los dos grupos de estudio en la preprueba de conceptos.

\begin{tabular}{lll}
\hline Medida & $G E$ & $G C$ \\
\hline Media & 4,57 & 4,71 \\
Desviación estándar & 0,38 & 0,56 \\
\hline
\end{tabular}

Tabla 4. Resultados de la preprueba sobre los conceptos y principios de la cinemática bidimensional.

El valor calculado para el parámetro de la prueba t fue de 0,946 , que es menor al mostrado en la tabla de la distribución t de Student para los valores de referencia. Esto permitió concluir que, antes de la fase instruccional, no existía diferencia estadísticamente significativa entre las calificaciones medias de los grupos en la prueba sobre conceptos y principios de la cinemática bidimensional. En otras palabras, se encontró correspondencia entre los niveles de comprensión de los principios de la cinemática bidimensional de cada uno de los grupos.

\subsection{Resultados de la fase post-instruccional.}

Para efectos de realizar la prueba t-Student, la primera hipótesis se transformó en las siguientes hipótesis estadísticas:

$\mathrm{H}_{1}$ : El GE y el GC difieren entre sí de manera significativa respecto a la puntuación media obtenida en la posprueba del test para diagnosticar la actitud de los estudiantes hacia la ciencia.

$\mathrm{H}_{0}^{\prime}$ : El GE y el GC no difieren entre sí de manera significativa respecto a la puntuación media obtenida en la posprueba del test para diagnosticar la actitud de los estudiantes hacia la ciencia.

Como puede verse en la tabla 5, el GE obtuvo una media más alta que la alcanzada por el GC. Asimismo, los alumnos que siguieron la secuencia didáctica mediada por el LV obtuvieron puntajes más agrupados en torno a la media.

\begin{tabular}{llll}
\hline Medida & GE & GC & $\Delta$ \\
\hline Media & 60,76 & 51,00 & $+9,76$ \\
Desviación estándar & 2,81 & 4,02 & $-1,21$
\end{tabular}

Tabla 5. Resultados de los grupos experimental y de control en el postest de Penichet y Mato.

Con estos resultados se realizó la prueba t de Student, la cual arrojó un valor de 9,415. Este resultado es considerablemente mayor al mostrado en la tabla de la distribución t de Student 
para los valores de referencia. Este valor sigue siendo mayor al mostrado en la tabla, inclusive para un nivel de confianza de 0,005 y el mismo número de grados de libertad. Entonces, se rechaza la hipótesis nula $\left(\mathrm{H}_{0}^{\prime}\right)$ y se acepta la primera hipótesis de investigación $\left(\mathrm{H}_{1}\right)$.

Asimismo, la segunda hipótesis de estudio, se transformó en las siguientes hipótesis estadísticas, para llevar a cabo el análisis paramétrico mediante la prueba t:

$\mathrm{H}_{2}$ : El GE y el GC difieren entre sí de manera significativa respecto a la puntuación media obtenida en la posprueba estandarizada sobre los conceptos de la cinemática bidimensional.

$\mathrm{H}_{0}{ }_{0}$ : El GE y el GC no difieren entre sí de manera significativa respecto a la puntuación media obtenida en la posprueba estandarizada sobre los conceptos de la cinemática bidimensional.

La siguiente tabla sintetiza los resultados de ambos grupos en la posprueba estandarizada sobre los conceptos y principios de la cinemática bidimensional:

\begin{tabular}{llll}
\hline & Grupo experimental & Grupo de control & $\Delta$ \\
\hline Media & 9,36 & 8,90 & $+0,46$ \\
Desviación estándar & 0,49 & 0,78 & $-0,32$ \\
\hline
\end{tabular}

Tabla 4. Resultados del GE y el GC en la posprueba sobre los conceptos de la cinemática bidimensional.

El GE obtuvo una media más alta y, además, los puntajes fueron menos dispersos que los obtenidos por los alumnos del GC. El cálculo de la prueba t dio un resultado de 2,352 que es mayor al que se muestra en la tabla de la distribución t de Student para los valores de referencia. Por lo tanto la hipótesis nula $\left(\mathrm{H}_{0}{ }^{\prime \prime}\right)$ es rechazada y se acepta la segunda hipótesis de estudio $\left(\mathrm{H}_{2}\right)$.

Finalmente, la tercera hipótesis de investigación se transformó en las siguientes hipótesis estadísticas, con el propósito de realizar la prueba t de Student:

$\mathrm{H}_{3}$ : El GE y el GC difieren entre sí de manera significativa respecto a la media obtenida en la posprueba de solución de problemas relacionados con la cinemática bidimensional.

$\mathrm{H}_{0}{ }^{\prime \prime}$ : El GE y el GC no difieren entre sí de manera significativa respecto a la media obtenida en la posprueba de solución de problemas relacionados con la cinemática bidimensional.

El GC obtuvo una ventaja de $+0,24$ en su puntuación media y una diferencia de $-0,46$ en la desviación estándar, en relación con los resultados del GE. El cálculo de la prueba t dio un resultado de 1,580. Este valor es menor al presentado en la tabla de la distribución t de Student para los valores de referencia. Por lo tanto se rechaza la tercera hipótesis de estudio $\left(\mathrm{H}_{3}\right)$ y se acepta la hipótesis nula $\left(\mathrm{H}_{0}{ }^{\prime \prime}\right)$.

\subsection{Análisis de resultados.}

Los resultados reportan que los alumnos que desarrollaron la estrategia de aprendizaje por descubrimiento mediado por LV tuvieron un mejor desempeño tanto en la dimensión actitudinal como la dimensión cognitiva. Esto guarda concordancia con los resultados de estudios como los de Amaya (2009), Bayrak (2008) y Sierra (2005). Retomando la pregunta general, ¿Cuál es el impacto del LV en el aprendizaje por descubrimiento de la cinemática 
bidimensional en estudiantes de décimo grado de Educación Media?, los resultados permiten concluir que el uso del LV tiene una incidencia significativamente positiva en la actitud y en la comprensión de los alumnos. Así, una estrategia de aprendizaje mediada por el uso de LV resulta más eficaz que una basada en enseñanza expositiva.

\section{DISCUSIÓN.}

\subsection{Hallazgos.}

Los resultados reportan que los estudiantes que emplearon el laboratorio virtual mostraron mayor motivación que quienes siguieron una estrategia de enseñanza tradicional. En suma, en relación con el primer objetivo del estudio y dando respuesta a la primera pregunta que orientó la investigación se establece que, para el nivel de Educación Media, la utilización del software de laboratorio virtual tiene una incidencia significativamente alta en el desarrollo de una buena actitud hacia el estudio de las asignaturas del área de ciencias naturales.

Los alumnos que utilizaron el laboratorio virtual mostraron un nivel de comprensión más alto que aquellos que siguieron un procedimiento tradicional. En relación al segundo objetivo del estudio y a la segunda pregunta de la investigación, se encontró que el uso del laboratorio virtual influye positivamente en el mejoramiento del nivel de comprensión de los conceptos y principios de la cinemática bidimensional. Asimismo, en relación al tercer objetivo del estudio y a la tercera pregunta de investigación, se concluye que el uso de laboratorio virtual como estrategia exclusiva de aprendizaje, permite desarrollar algunas habilidades para resolver problemas pero no reporta una ventaja significativa en relación con una estrategia de enseñanza tradicional.

\subsection{Recomendaciones.}

El software de laboratorio virtual puede ser utilizado para identificar las ideas previas en relación con los fenómenos físicos estudiados. De igual manera permite realizar un abordaje de los eventos físicos desde una perspectiva cualitativa al ser utilizado como una herramienta mediadora en el proceso de aprendizaje de la cinemática. Esta herramienta se constituye en un medio de evaluación, un instrumento para la recuperación y la profundización de los estudiantes.

Como futuras investigaciones se sugieren estudios sobre el impacto del laboratorio virtual en otros ámbitos de la física, así como en otras asignaturas de ciencias. Asimismo es importante valorar la incidencia de esta herramienta en otros niveles educativos. Se recomiendan estudios cualitativos que permitan ampliar la visión sobre el tema. Finalmente, es importante comprender la influencia de diversos factores en la eficacia del software.

En cuanto a las debilidades del estudio, se debe tener en cuenta que los sujetos pertenecen a grupos intactos, por lo que las conclusiones de la investigación no pueden ser transferidas a otros contextos. Otro factor que influye en el estudio es la heterogeneidad en el nivel de habilidad para utilizar el computador, en el grado de razonamiento lógico, en el rendimiento y 
en los estilos de aprendizaje. De otro lado, la rigidez del enfoque cuantitativo limita la comprensión del fenómeno.

\section{REFERENCIAS}

AMAYA, G. (2008). La simulación computarizada como instrumento del método en el proceso de enseñanza y aprendizaje de la física, desde la cognición situada: ley de Ohm. Revista electrónica Actualidades Investigativas en Educación, 8 (1). Recuperado de http://revista.inie.ucr.ac.cr/articulos/1-2008/archivos/ohm.pdf

AMAYA, G. (2009). Laboratorios reales versus laboratorios virtuales, en la enseñanza de la física [Versión electrónica]. El hombre y la máquina, 21 (33), 82-95.

BAYRAK, C. (2008). Effects of computer simulations programs on university students' achievements in physics. Turkish Online Journal of Distance Education, 9 (4). Recuperado de http://tojde.anadolu.edu.tr/tojde32/pdf/article_3.pdf

BECERRA, F. (2005). Aprendizaje en colaboración mediado por simulación en computador. Efectos en el aprendizaje de procesos termodinámicos. [Versión electrónica], Revista de estudios sociales, 20, 13-26.

CATALÁN, L. C., SERRANO, G. M., Y CONCARI, S. B. (2010). Construcción de significados en alumnos de nivel básico universitario sobre la enseñanza de física con empleo de software [Versión electrónica]. Revista mexicana de investigación educativa, 15 (46), 873-893.

CASADEI, L., CUICAS, M., DEBEL, E. Y ÁLVAREZ, Z. (2008). La simulación como herramienta de aprendizaje en física. Actualidades Investigativas en Educación, 8, (2). Recuperado de http://revista.inie.ucr.ac.cr/articulos/2-2008/archivos/fisica.pdf

CHOU, C. (1998). The effectiveness of using multimedia computer simulations coupled with social constructivist pedagogy in a college introductory physics classroom. Disertación doctoral no publicada. Graduate School of Education, Columbia University.

DEBEL, E., CUICAS, M., CASADEI, L., Y ÁLVAREZ, Z. (2009). Experimento real y simulación como herramientas de apoyo para lograr aprendizajes significativos en la asignatura Laboratorio de Física II. Multiciencias, 9, (1), 80-88.

ESCRIBANO, A. (2008). El aprendizaje basado en problemas: una propuesta metodológica en educación superior. Madrid, España: Narcea.

ESQUEMBRE, F. (2005). Creaciones de simulaciones interactivas en Java. Madrid, España: Pearson Educación.

FOGLIATI, P., CATALÁN, L. C. Y CONCARI, S. B. (2004, Octubre). Dificultades procedimentales en la resolución de problemas con simulaciones computarizadas. Presentado en el 70 Simposio de Investigación en Educación en Física. Santa Rosa, La Pampa, Argentina. 
GARCÍA, A. Y GIL, M. (2006). Entornos constructivistas de aprendizaje basados en simulaciones interactivas. Revista Electrónica de Enseñanza de las Ciencias, 5 (2). Recuperado de http://www.saum.uvigo.es/reec/volumenes/volumen5/ART6 Vol5 N2.pdf

GIORGI, S., CÁMARA, C. Y KOFMAN, H. (2004, Octubre). El uso de la computadora en las modalidades de simulación y adquisición de datos para el estudio del campo magnético en un solenoide por el que circula corriente continua. Presentado en el 70 Simposio de Investigación en Educación en Física. Santa Rosa, La Pampa, Argentina.

OLIVERO, J. Y CHIRINOS, J. (2007). Estrategias interactivas basadas en las nuevas tecnologías de la información aplicadas en física [Versión electrónica]. Multiciencias, 7 (2), 207-217

PENICHET, A. Y MATO, M. C. (1999). Las actitudes del alumnado de secundaria hacia las ciencias experimentales. Alambique, Didáctica de las Ciencias Experimentales, 22, 9-16.

KELLY, G. A. (1955). The psychology of personal constructs. Nueva York, Estados Unidos: Norton.

KOFMAN, H. A., CATALÁN, L. C. Y CONCARI, S. B. (2004, Marzo). A training distance course on the use of simulations for physics teaching. Presentado en el World Congress on Engineering and Technology Education. Guarujá, São Paulo, Brasil.

KOWALSKI, L. (1985). A comment about the definition of simulation. Journal of Computer in Mathematics and Science Teaching, 4, 50-51.

RODRÍGUEZ, D., MENA, D. Y RUBIO, C. (2009). Uso de software de simulación en la enseñanza de la Física. Una aplicación en la carrera de Ingeniería Química [Versión electrónica], Tecnología, Ciencia, Educación, 24, (2), 127-136.

SIERRA, J. L. (2000). Informática y enseñanza de las ciencias. En Perales, F. J. y Cañal, P. (Eds.), Didáctica de las Ciencias Experimentales (pp. 339 - 360) Alcoy, España: Marfil.

SIERRA, J. L., (2005). Estudio de la influencia de un entorno de simulación por ordenador en el aprendizaje por investigación de la Física en Bachillerato. Madrid, España: Gobierno de España, Ministerio de Educación y Ciencia.

TÜIZÜZ, C. (2010). The effect of the virtual laboratory on students' achievement and attitude in chemistry. International Online Journal of Educational Sciences, 2 (1). Recuperado de http://www.iojes.net/userfiles/Article/IOJES 167.pdf

\section{Para citar este artículo:}

ANGULO, G. A., VIDAL, L. O. \& GARCÍA, G. (2012). Impacto del laboratorio virtual en el aprendizaje por descubrimiento de la cinemática bidimensional en estudiantes de educación media. EDUTEC, Revista Electrónica de Tecnología Educativa, 40. Recuperado el dd/mm/aa de http://edutec.rediris.es/Revelec2/Revelec40/impacto laboratorio virtual aprendizaje descubri miento cinematica bidimensional educacion media.html 